\title{
The type of sugar moiety is a major determinant of the small intestinal uptake and subsequent biliary excretion of dietary quercetin glycosides
}

\author{
Ilja C. W. Arts ${ }^{1 *}$, Aloys L. A. Sesink ${ }^{2}$, Maria Faassen-Peters ${ }^{3}$ and Peter C. H. Hollman ${ }^{1}$ \\ ${ }^{1}$ RIKILT - Institute of Food Safety, Wageningen University and Research Centre, Wageningen, The Netherlands \\ ${ }^{2}$ Department. of Pharmacology and Toxicology, Nijmegen Centre for Molecular Life Sciences, Nijmegen, \\ The Netherlands \\ ${ }^{3}$ Small Animal Research Centre, Wageningen University, Wageningen, The Netherlands
}

(Received 22 October 2003 - Revised 26 January 2004 - Accepted 8 February 2004)

\begin{abstract}
Quercetin is an important dietary flavonoid with putative beneficial effects in the prevention of cancer and CVD. The in vivo bioactivity of quercetin depends on its bioavailability, which varies widely between foods. We used an in situ rat intestinal perfusion model to study whether differential small intestinal hydrolysis of the sugar moiety of five naturally occurring quercetin glycosides determines the small intestinal uptake and subsequent biliary excretion of quercetin. After $30 \mathrm{~min}$ perfusion, a decrease of intact quercetin glycoside in perfusate was observed for quercetin-3-O-ß-glucoside (20.9 (SEM 1.4) $\mu \mathrm{mol} / \mathrm{l}$ ) and quercetin-4'-O-ß-glucoside (23.5 (SEM 1.6$) \mu \mathrm{mol} /$ 1 ), but not of quercetin-3-O- $\beta$-galactoside, quercetin-3- $O$ - $\beta$-rhamnoside and quercetin-3- $O$ - $\alpha$-arabinopyranoside. Appearance of free quercetin in perfusate and conjugated quercetin metabolites (quercetin, isorhamnetin, and tamarixetin) in portal and peripheral plasma and bile were also significantly greater after treatment with quercetin-3-O-ß-glucoside or quercetin-4'-O-B-glucoside compared with any of the other glycosides. Thus, the type of sugar moiety is a major determinant of the small intestinal absorption of quercetin glycosides, but the position $\left(3\right.$ or $\left.4^{\prime}\right)$ of the glucose moiety does not further influence absorption. The poor bioavailability of important dietary quercetin glycosides has implications for their in vivo bioactivities.
\end{abstract}

Flavonoid: Quercetin glycoside: Intestinal absorption: Lactase phlorizin hydrolase: Bile

Quercetin is an important dietary flavonoid with antioxidant, anti-inflammatory, antiproliferative and anticarcinogenic properties (Nijveldt et al. 2001). Because of its putative beneficial effects in the prevention of cancer and CVD, quercetin is used as a food supplement and a promising ingredient of so-called functional foods (Verhoeyen et al. 2002). The in vivo bioactivity of quercetin is determined by concentrations at target sites, and thus by the degree of intestinal absorption from the diet, by metabolism, and by excretion into bile, urine and faeces, i.e. its bioavailability. The bioavailability of quercetin from different foods varies widely (Hollman et al. 1997), and it is unclear what causes this. In plant foods, quercetin occurs almost exclusively bound to one or more sugar molecules, so-called quercetin- $\beta$-glycosides. The dominant type of glycoside varies between foods. Apple, for example, contains predominantly galactosides, rhamnosides and arabinosides, while onion contains mainly glucosides (Hollman \& Arts, 2000). We hypothesise that the sugar moiety is an important determinant of the bioavailability of quercetin from foods. There is some evidence in the literature to support this hypothesis: while quercetin-3-glucoside and quercetin-4'-glucoside were rapidly absorbed in human subjects, quercetin-3-rutinoside (a diglycoside predominant in tea) was not (Hollman et al. 1997; Olthof et al. 2000).

Because $\beta$-glycosides are resistant to hydrolysis by pancreatic enzymes, absorption of quercetin- $\beta$-glycosides was at first thought to occur only after hydrolytic enzymes from the colonic microflora had released the aglycone. However, Hollman et al. (1997) showed that quercetin appeared in plasma only $30 \mathrm{~min}$ after ingestion of quercetin glucoside-rich foods, pointing at absorption in the small intestine. At first, the mechanisms involved in small intestinal uptake and transport of intact quercetin glycosides were thought to be mediated by Na-dependent glucose transporter 1 , followed by cytosolic B-glucosidase-mediated hydrolysis of the sugar moiety (Hollman et al. 1999;

Abbreviations: AUC, area under the concentration-time curve; LPH, lactase phlorizin hydrolase; Q3arabinoside, quercetin-3- $O$ - $\alpha$-arabinopyranoside; $\mathrm{Q} 3$ galactoside, quercetin-3-O-ß-galactoside; Q3glucoside, quercetin-3-O-B-glucoside; Q3rhamnoside, quercetin-3-O-ß-rhamnoside; $\mathrm{Q} 4^{\prime}$ glucoside, quercetin-4'-O-ß-glucoside.

* Corresponding author: Dr Ilja C. W. Arts, fax + 31317417717 , email ilja.arts@wur.nl 
Day \& Williamson, 2001). Although these pathways may contribute to the absorption of quercetin glycosides, it is becoming increasingly clear that lactase phlorizin hydrolase (LPH) is the main determinant of uptake (Day et al. 2003; Sesink et al. 2003). LPH is a mammalian $\beta$-glycosidase present in the brush border membrane of the small intestine; it is thought to play a role in the rapid in vivo uptake of these quercetin glucosides (Day et al. 2000b). In vitro studies with purified LPH from lamb intestine showed that several flavonoid glucosides were indeed substrates for this enzyme (Day et al. 2000b). We recently demonstrated that during perfusion of the rat small intestine with quercetin-3-glucoside, inhibition of LPH caused a $67 \%$ reduction in quercetin-3-glucoside hydrolysis, concomitant with a $75 \%$ reduction in plasma quercetin concentration (Sesink et al. 2003). The substrate-specificity of LPH for hydrolysis of different quercetin glycosides may vary and thus contribute to their varying bioavailability.

Upon absorption, the biological activity of quercetin can be modified by conjugation reactions, such as glucuronidation and/or sulfation, which take place in the cells of the intestine and liver (Rice-Evans et al. 1996; Day et al. $2000 a$ ). In addition, hydroxyl groups of the catechol-ring can be methylated by the enzyme catechol- $O$-methyltransferase. The main $O$-methylated derivative of quercetin in plasma of human subjects and rats is isorhamnetin (quercetin-3'-methyl ether; Hollman \& Arts, 2000; Stahl et al. 2002). Bile is considered an important excretion route for flavonoids. However, firm evidence to support this contention is limited, and it is unclear whether the excretion pattern differs between quercetin glycosides. In rats that were fed a high-quercetin diet for $14 \mathrm{~d}$, the concentration of quercetin in bile was sevenfold higher than that in plasma, and an additional metabolite, tamarixetin (quercetin-4'-methyl ether), was detected (Manach et al. 1998).

Thus far, the small intestinal uptake of different quercetin glycosides and their subsequent biliary excretion as conjugated metabolites has not been systematically investigated. In the present study, we used an in situ rat intestinal perfusion model to study whether the appearance of quercetin and its methylated derivatives in plasma and bile is determined by small intestinal hydrolysis of five major quercetin monoglycosides (4'-glucoside, 3-glucoside, 3-galactoside, 3-rhamnoside and 3-arabinoside).

\section{Materials and methods}

\section{Chemicals}

Quercetin-3-O-B-glucoside (Q3glucoside), quercetin-4'- $O$ $\beta$-glucoside (Q4'glucoside), quercetin-3-O-B-galactoside (Q3galactoside), quercetin-3-O-ß-rhamnoside (Q3rhamnoside), quercetin-3- $O$ - $\alpha$-arabinopyranoside (Q3arabinoside) and tamarixetin (quercetin-4'-methyl ether) were purchased from Extrasynthese (Genay, France). The identity of Q3arabinoside was confirmed using nuclear magnetic resonance because it was previously sold wrongly labelled as quercetin-3-O- $\alpha$-arabinofuranoside (avicularin; Lommen et al. 2000). Isorhamnetin (quercetin-3'-methyl ether) was purchased from Roth (Karlsruhe, Germany).
Quercetin and B-glucuronidase-sulfatase (Helix pomatia, G1512) were obtained from Sigma (St Louis, MO, USA). All other chemicals used were of analytical grade. A mix of quercetin glucuronides used in the present study was kindly provided by Karen O'Leary (Institute of Food Research, Norwich, Norfolk, UK).

\section{Animals and diets}

The experimental protocol was approved by the Animal Welfare Committee of Wageningen University, Wageningen, The Netherlands. Male outbred Wistar rats (Harlan, Horst, The Netherlands; specific pathogen free, $n 31$ ), mean body weight 208 (SD 8.1) g, were housed individually in a room with controlled temperature $\left(22-24^{\circ} \mathrm{C}\right)$ and relative humidity $(50-60 \%)$ and a $12 \mathrm{~h}$ light-dark cycle (lights on 06.00 hours). For three consecutive days before the experiment, all rats were fed a commercially available soyabean-free semipurified diet (composition $(\mathrm{g} / \mathrm{kg})$ : dextrose 540, cellulose 50, maize starch 100, casein 200, maize oil 50, standard AIN-76 vitamin and mineral mix 5; Hope Farms, Woerden, The Netherlands). After $3 \mathrm{~d}$ plasma quercetin levels in all rats were below the limit of detection $(11 \mathrm{nmol} / \mathrm{l})$.

\section{In situ perfusion studies}

After limiting the feed intake overnight to $5 \mathrm{~g}$, rats were anaesthetised by inhalation of isoflurane, using $\mathrm{NO}-\mathrm{O}_{2}$ $(1: 1, \mathrm{v} / \mathrm{v})$ as carrier. First, the abdominal cavity was opened and the bile duct and portal vein were cannulated. Then two cannulas (internal diameter $1.52 \mathrm{~mm}$ ) were inserted in the small intestine (duodenum, jejunum and ileum), distal of the entrance of the bile duct in the duodenum and proximal of the caecum. The contents of the cannulated segment were removed before inserting the most distal cannula by flushing gently with $60 \mathrm{ml}$ saline $(9 \mathrm{~g}$ $\mathrm{NaCl} / 1,37^{\circ} \mathrm{C}$ ). Perfusion was started by injecting $10 \mathrm{ml}$ test solution $\left(37^{\circ} \mathrm{C}\right)$ into the cannulated segment and connecting the intestine immediately to a single-pass perfusion system. Constant perfusion took place at a flow rate of $1 \mathrm{ml} / \mathrm{min}$ at $37^{\circ} \mathrm{C}$, using a potassium phosphate buffer (5 mM, pH 6.7) containing (mmol/l): $\mathrm{NaCl} 100, \mathrm{KCl} 20$, $\mathrm{CaCl}_{2} 2, \mathrm{MgCl}_{2}$ 2, sodium taurocholate 0.5.

The perfusion buffer was supplemented with $50 \mu \mathrm{mol}$ Q3glucoside, Q4'glucoside, Q3galactoside, Q3rhamnoside or Q3arabinoside/l. In control experiments where these glycosides were incubated in perfusion fluid at $37^{\circ} \mathrm{C}$ for $60 \mathrm{~min}$, there was no disappearance of the glycosides and no free aglycone formed, indicating that they were stable under the experimental conditions. Perfusion was stopped after $30 \mathrm{~min}$, and in the last minute $1 \mathrm{ml}$ samples were taken from the perfusion fluid at the end of the cannulated segment and at the inlet of the perfused segment (for the $t$ 0 min sample). In a pilot experiment where the small intestine was perfused for 60 min with Q3glucoside, steadystate plasma and perfusion buffer concentrations were reached at $t 30 \mathrm{~min}$ (results not shown). Effluent samples were stabilised with acetic acid (10 mM, pH 4.9) and ascorbic acid ( $1 \mathrm{~g} / \mathrm{l}$, final concentrations). Volume changes in perfusion buffer during perfusion were recorded 
by weighing. Perfusion buffer volumes recovered did not differ significantly between groups (one-way ANOVA, $P=0.97$ ) and were 33.2 (Q3glucoside), 32.8 (Q4' glucoside), 34.4 (Q3galactoside), 33.5 (Q3rhamnoside) and 33.3 (Q3arabinoside). Because volume changes were due not only to water absorption during intestinal passage, but also to sampling, some spilling, and injecting approximately $10 \mathrm{ml}$ into the intestine before starting perfusion, these data could not be used to correct effluent sample concentrations of the glycosides. Because hydrolysis and absorption of Q3rhamnoside was almost negligible (indicated by the absence of free quercetin and quercetin metabolites in perfusion fluid and plasma), the $6 \%$ increase in perfusion buffer concentration of Q3rhamnoside during intestinal passage was attributed to water absorption. Thus, all $t 30$ perfusion buffer data presented here have been reduced by $6 \%$. Bile was collected in two portions (from $0-15$ and from 15-30 min). Before the experiment, and at 15 and $30 \mathrm{~min}$, blood was drawn from the cannulated portal vein, and after the experiment peripheral blood was drawn from the inferior vena cava. Blood was collected in EDTA-tubes and plasma was subsequently prepared by centrifuging for $10 \mathrm{~min}$ at $2000 \mathrm{~g}$ and $4^{\circ} \mathrm{C}$. All samples were stored at $-80^{\circ} \mathrm{C}$ before analysis.

\section{Preparation of samples}

Samples were analysed using a validated HPLC-coularray method (Arts et al. 2003). In plasma and bile, quercetin and its methylated metabolites isorhamnetin and tamarixetin only occur as glucuronidated and/or sulfated conjugates, so enzymatic hydrolysis with B-glucuronidase-sulfatase is required. In the remainder of the present paper, when referring to quercetin, isorhamnetin or tamarixetin concentrations in plasma or bile, the total concentration after hydrolysis of the conjugates is meant. Perfusion buffer effluents were only analysed without previous treatment with B-glucuronidase-sulfatase. Plasma $(50 \mu \mathrm{l})$ was incubated with $0.25 \mathrm{mg} \beta$-glucuronidase-sulfatase (Helix pomatia, $125 \mathrm{U} \quad \beta$-glucuronidase and $7 \mathrm{U}$ sulfatase) in $20 \mu \mathrm{l} 0.5 \mathrm{M}$-sodium acetate buffer $(\mathrm{pH} 4.9)$ with $6 \mathrm{~g}$ ascorbic acid/l. After incubating for $2 \mathrm{~h}$ at $37^{\circ} \mathrm{C}$, samples were deproteinised with 2 vols acetonitrile and thoroughly mixed. Then, 1 vol. $o$-phosphoric acid ( $200 \mathrm{ml} / \mathrm{l}$, containing $3 \mathrm{~g}$ ascorbic acid/l) was added. Samples were centrifuged for $10 \mathrm{~min}$ at $10000 \mathrm{~g}$ and $4{ }^{\circ} \mathrm{C}$ and analysed with HPLC. The analysis of bile was similar to that of plasma: $35 \mu \mathrm{l}$ bile was incubated with $0.25 \mathrm{mg}$ $\beta$-glucuronidase-sulfatase in $15 \mu \mathrm{l} 0.5 \mathrm{M}$-sodium acetate buffer ( $\mathrm{pH} 4.9$ ) with $6 \mathrm{~g}$ ascorbic acid/l. After incubation for $1 \mathrm{~h}$ at $37^{\circ} \mathrm{C}$, samples were deproteinised with 2 vols acetonitrile and 1 vol. $o$-phosphoric acid $(200 \mathrm{ml} / \mathrm{l})$. All analyses were performed in simplo (single).

\section{HPLC analysis}

Merck Hitachi L6000A and L6200 pumps (Hitachi Ltd, Tokyo, Japan) were equipped with a Gilson 234 autosampler (Gilson, Villiers-le-Bel, France) and a coulometric detector (Coularray detector model 5600; ESA, Chelmsford, MA, USA) set at 100, 250, and $500 \mathrm{mV} \mathrm{(Pd} \mathrm{as}$ reference). Separation of a $20 \mu \mathrm{l}$ sample was performed on a Chromolith RP-18e column $(100.0 \times 4.6 \mathrm{~mm}$; Merck, Darmstadt, Germany) protected by a NewGuard RP18 guard column $(15.0 \times 3.2 \mathrm{~mm}, 7 \mu \mathrm{m}$; Perkin Elmer, Norwalk, CT, USA). The columns were maintained at $30^{\circ} \mathrm{C}$ with an ESA column heater. The solvents for gradient elution were $50 \mathrm{ml}$ acetonitrile/l (solvent A) and $700 \mathrm{ml}$ acetonitrile/l (solvent B) in citrate buffer $(25 \mathrm{~mm}, \mathrm{pH}$ $3.7)$. The following gradient, at a flow rate of $2.5 \mathrm{ml} / \mathrm{min}$, was used: $0 \cdot 0-12.0 \mathrm{~min}$, linear gradient from 0 to $43 \%$ $\mathrm{B} ; 12.0-13.0 \mathrm{~min}$, linear gradient to $100 \% \mathrm{~B} ; 13.0-$ $14.5 \mathrm{~min}, 100 \% \mathrm{~B} ; 14.5-15.5 \mathrm{~min}$, linear gradient to $0 \%$ B. Total run time including equilibration was $17 \mathrm{~min}$.

\section{Statistical analyses}

Results are presented as mean values with their standard errors. One-way ANOVA followed by Bonferroni multiple comparison tests were used to test for statistically significant differences between treatment groups using the SPSS statistical package (SPSS for Windows, version 10.0.5; SPSS Inc., Chicago, IL, USA). The area under the concentration-time curve (AUC) for quercetin and isorhamnetin in portal vein plasma was calculated by the trapezoidal rule.

\section{Results}

After perfusing the rat small intestine for $30 \mathrm{~min}$ with solutions of $50 \mu \mathrm{mol}$ Q3glucoside, Q4'glucoside, Q3galactoside, Q3rhamnoside or Q3arabinoside/l, effluent concentrations of the intact glycoside were lower than concentrations at the inlet for the Q3glucoside and the Q4'glucoside groups only (Fig. 1). The decline in concentration was similar for Q3glucoside (20.9 (SEM 1.4) $\mu \mathrm{mol} / \mathrm{l})$ and for Q4' glucoside (23.5 (SEM 1.6) $\mu \mathrm{mol} / \mathrm{l}$ ), but both differed significantly from changes observed in the other groups $(P<0 \cdot 05)$. At the same time, free quercetin appeared in the effluent of the Q3glucoside (2.9 (SEM $0.5) \mu \mathrm{mol} / \mathrm{l}$ ) and at a slightly higher concentration in the Q4'glucoside (6.1 (SEM 1.0) $\mu \mathrm{mol} / \mathrm{l})$ group $(P<0.05)$, but only marginally in the other groups (Fig. 1).

Fig. 2 shows that during perfusion, there was an increase in portal vein plasma concentration of total quercetin (after enzymatic hydrolysis of the glucuronide and sulfate conjugates) from 0 (limit of detection $11 \mathrm{nmol} / \mathrm{l}$ ) to about $4 \mu \mathrm{mol} / \mathrm{l}$ at $15 \mathrm{~min}$ and about $5 \mu \mathrm{mol} / 1$ at $30 \mathrm{~min}$ in all animals of the Q3glucoside and the Q4'glucoside groups (Fig. 2(A)). The AUC for these groups were similar $(90 \cdot 2$ (SEM 9.0) and 101.3 (SEM 16.6) $\mu \mathrm{mol} \cdot \mathrm{min} / \mathrm{l}$ respectively), but differed significantly $(P<0 \cdot 05)$ from the other experimental groups where only trace amounts of quercetin were detected (up to 0.6 (SEM 0.1) $\mu \mathrm{mol} / \mathrm{l}$ for Q3galactoside). A similar pattern was seen for the $3^{\prime}$-methylated quercetin metabolite isorhamnetin (Fig 2(B)). No isorhamnetin (limit of detection $14 \mathrm{nmol} / \mathrm{l}$ ) could be detected in plasma of the Q3galactoside, Q3rhamnoside and Q3arabinoside animals. Peripheral plasma was collected at 30 min only. Quercetin and isorhamnetin concentrations in peripheral plasma were of the same order of magnitude 


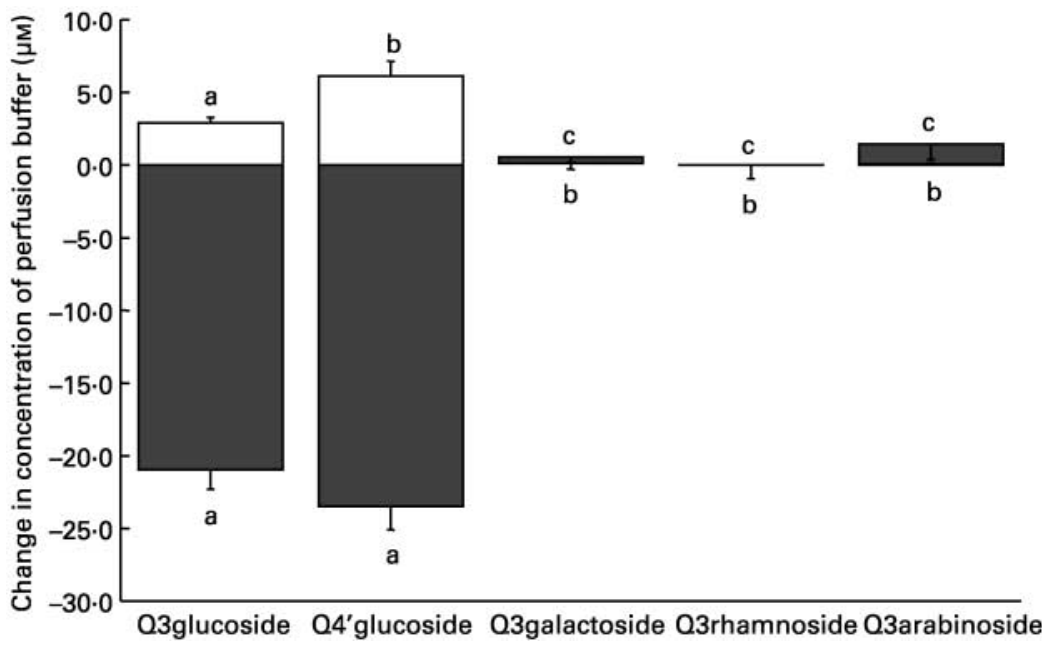

Fig. 1. Change in concentration of quercetin glycosides ( $\square$ ) and quercetin aglycone ( $\square$ ) in perfusion buffer between 0 and $30 \mathrm{~min}$ in situ perfusion of rat small intestine with $50 \mu \mathrm{mol}$ quercetin glycosides/l. Q3glucoside, quercetin-3-O-B-glucoside; Q4'glucoside, quercetin-4'-O-Bglucoside; Q3galactoside, quercetin-3-O-B-galactoside; Q3rhamnoside, quercetin-3-O- $\beta$-rhamnoside; Q3arabinoside, quercetin-3-O- $\alpha$-arabinopyranoside. For details of procedures, see p. 843. Values are means with their standard errors shown by vertical bars ( $n 6 ; n 7$ for Q3glucoside). ${ }^{a, b, c}$ Mean values with unlike superscript letters (above $x$-axis for quercetin aglycone and below $x$-axis for quercetin glycosides) were significantly different between groups $(P<0.05)$.

as the portal vein concentrations at $30 \mathrm{~min}$ for all five experimental groups (Table 1).

After absorption, quercetin metabolites were rapidly excreted into the bile. In addition to quercetin and
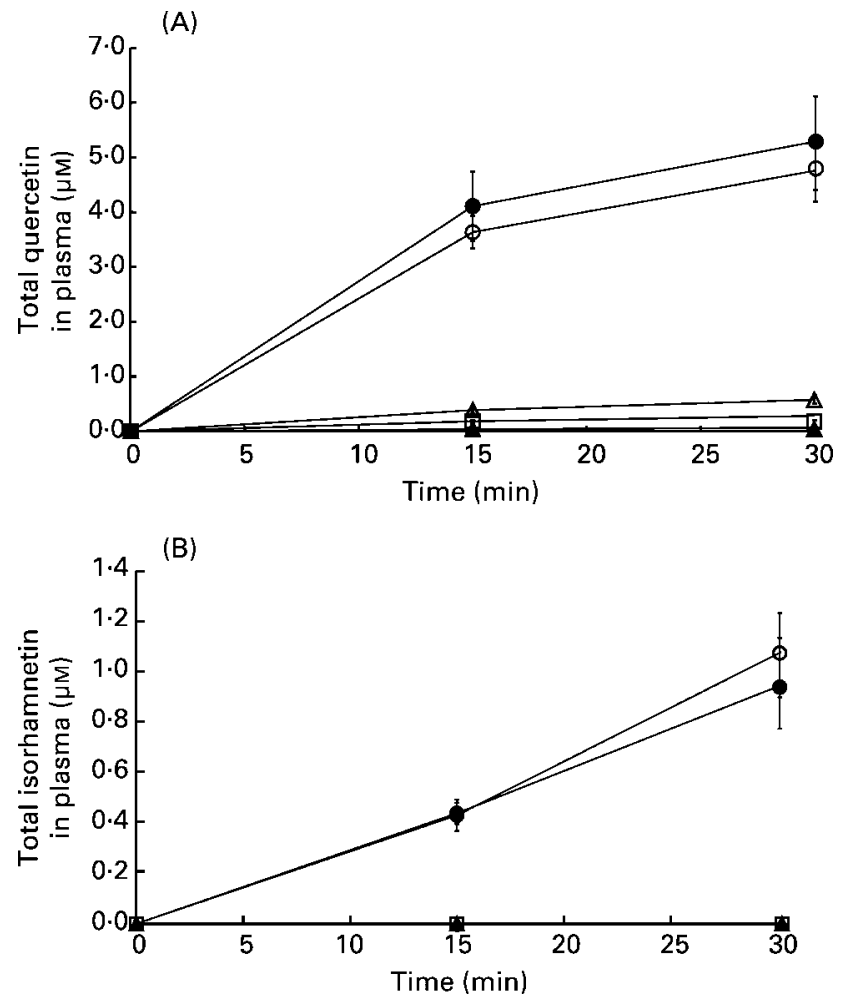

Fig. 2. Portal vein plasma concentrations of total quercetin (A) (limit of detection $11 \mathrm{nmol} / \mathrm{l}$ ) and isorhamnetin (B) (limit of detection 14 $\mathrm{nmol} / \mathrm{l})$ measured by HPLC after enzymatic hydrolysis during in situ perfusion of rat small intestine with $50 \mu \mathrm{mol}$ quercetin-3-O-B-glucoside $(O)$, quercetin-4'-O-B-glucoside $(\bullet)$, quercetin-3-O-ß-galactoside $(\triangle)$, quercetin-3-O-B-rhamnoside $(\boldsymbol{\Lambda})$ or quercetin-3-O- $\alpha-$ arabinopyranoside $(\square) / /$. For details of procedures, see p. 843. Values are means with their standard errors shown by vertical bars ( $n 6 ; n 7$ for quercetin-3-O-ß-glucoside). isorhamnetin that were found in plasma, bile contained a second methylated metabolite: tamarixetin. Quercetin, isorhamnetin and tamarixetin were present in approximately equimolar concentrations in bile (Fig. 3); this did not vary between the first and second $15 \mathrm{~min}$ of sample collection (results not shown). In the sample obtained during the first $15 \mathrm{~min}$ of perfusion, the total concentration of quercetin metabolites was 49.6 (SEM 20.6) $\mu \mathrm{mol} / \mathrm{l}$ for the Q3glucoside and 34.0 (SEM 11.0) $\mu \mathrm{mol} / \mathrm{l}$ for the Q4'glucoside group. Concentrations for the other experimental groups did not exceed $5 \mu \mathrm{mol} / \mathrm{l}$ (results not shown). During the second $15 \mathrm{~min}$, concentrations measured were as great as 324.1 (SEM 64.7) (Q3glucoside) and $349 \cdot 3$ (SEM 47.2) $\mu \mathrm{mol} / \mathrm{l}$ (Q4'glucoside) (Fig. 3). The highest concentration of metabolites in the other three experimental groups was found after perfusion with Q3galactoside and amounted to 16.3 (SEM 2.4) $\mu \mathrm{M}$. Again, all individual metabolites and the total metabolite concentrations were similar for the Q3glucoside and Q4'glucoside group, but differed significantly from the other three groups $(P<0 \cdot 05)$.

Table 1. Peripheral plasma concentrations ( $\mu \mathrm{mol} / \mathrm{l})$ at $t=30 \mathrm{~min}$ of quercetin metabolites after enzymatic hydrolysis $\dagger$

\begin{tabular}{|c|c|c|c|c|c|c|}
\hline & \multicolumn{2}{|c|}{ Quercetin } & \multicolumn{2}{|c|}{ Isorhamnetin } & \multicolumn{2}{|c|}{ Total } \\
\hline & Mean & SEM & Mean & SEM & Mean & SEM \\
\hline Q3glucoside & $3 \cdot 8^{*}$ & 0.56 & $1 \cdot 2^{\star}$ & 0.19 & $5 \cdot 0^{*}$ & 0.73 \\
\hline Q4'glucoside & $4 \cdot 5^{\star}$ & 0.83 & $1 \cdot 1^{*}$ & 0.20 & $5 \cdot 6^{*}$ & 1.01 \\
\hline Q3galactoside & 0.5 & 0.10 & 0.0 & 0.02 & 0.6 & 0.12 \\
\hline Q3rhamnoside & 0.0 & 0.02 & $<$ LOD $\ddagger$ & - & 0.0 & 0.02 \\
\hline Q3arabinoside & 0.3 & 0.03 & $<\mathrm{LOD} \ddagger$ & - & 0.3 & 0.03 \\
\hline
\end{tabular}

Q3glucoside, querceitin-3-O-ß-glucoside; Q4'glucoside, quercetin-4'-O-ß-glucoside; Q3galactoside, quercetin-3-O-B-galactoside; Q3rhamnoside; quercitin-3-O-ß-rhamnoside; Q3arabinoside, quercetin-3-O- $\alpha$-arabinopyranoside; LOD, limit of detection.

*Mean values were significantly different from those of other quercetin glycoside groups: $P<0.05$.

†For details of procedures, see p. 843.

$\ddagger$ LOD $14 \mathrm{~nm}$. 


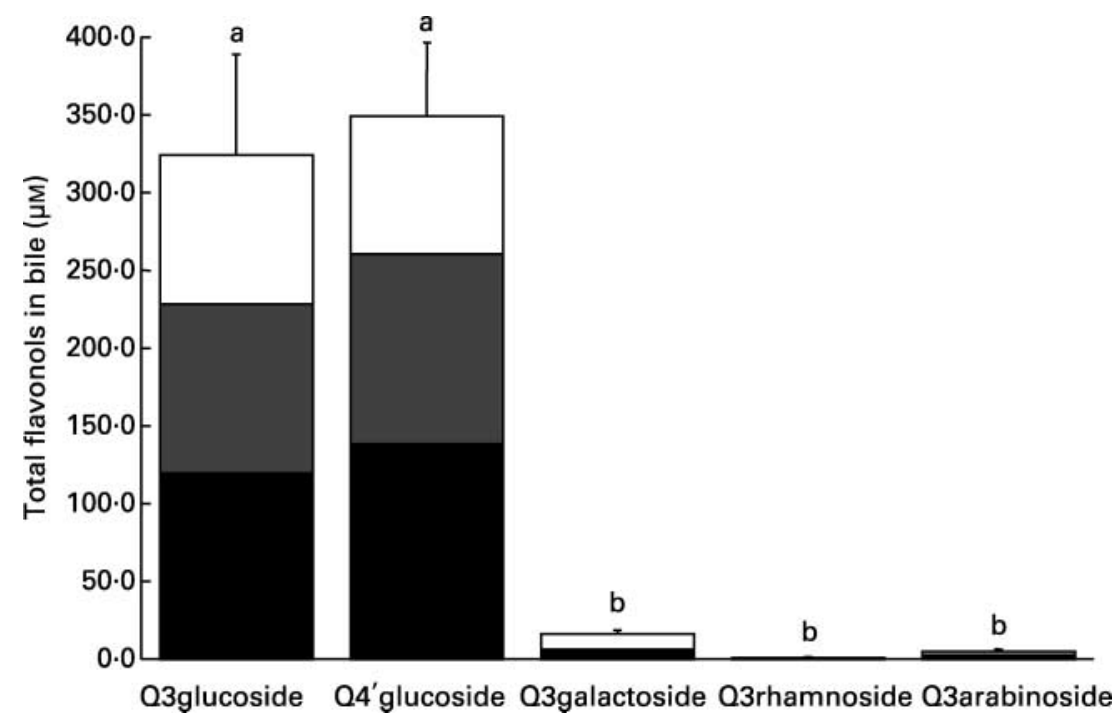

Fig. 3. Bile concentrations of quercetin ( $\square$ ), isorhamnetin $(\square)$ and tamarixetin ( $\square$ ) measured by HPLC after enzymatic hydrolysis in the $t$ 15$30 \mathrm{~min}$ samples collected during in situ perfusion of rat small intestine with $50 \mu \mathrm{mol}$ quercetin glycoside/l. Values are means with their standard errors shown by vertical bars ( $n 6 ; n 7$ for quercetin-3-O-B-glucoside). For details of procedures, see $p .843 .{ }^{a, b}$ Mean values with unlike superscript letters were significantly different between groups for total and individual metabolite concentrations $(P<0.05)$.

\section{Discussion}

The results of the present study suggest that the type of sugar, but not the position at which it is attached to the quercetin molecule, is the major determinant of the small intestinal absorption of quercetin in rats. Both Q3glucoside and $\mathrm{Q}^{\prime}$ glucoside were hydrolysed by $\beta$-glycosidases present in the small intestine and were rapidly absorbed. After perfusing the small intestine for $30 \mathrm{~min}$ with a $50 \mu \mathrm{mol}$ glucoside/l, peripheral plasma metabolite concentrations were as great as $5.0 \mu \mathrm{mol} / 1$ for the 3 -glucoside and $5.6 \mu \mathrm{mol} / \mathrm{l}$ for the $4^{\prime}$-glucoside. Portal vein plasma concentrations were slightly higher. In contrast, other glycosides were resistant to intestinal hydrolysis and only trace amounts of the metabolites of Q3galactoside, Q3rhamnoside or Q3arabinoside were detected in plasma. The data for perfusion buffer were in accordance with the plasma findings, which supports data suggesting that intestinal hydrolysis of the sugar moiety is a necessary step for absorption of quercetin glycosides to occur (Crespy et al. 2001; Day et al. 2003; Sesink et al. 2003).

The rapid and efficient uptake of Q3glucoside and Q4'glucoside has been reported before for human subjects (Olthof et al. 2000; Graefe et al. 2001), intact rats (Morand et al. 2000) and isolated or in situ perfused small intestine of rats (Spencer et al. 1999; Gee et al. 2000; Crespy et al. 2001; Day et al. 2003; Sesink et al. 2003). The absorption of Q3glucoside and Q4'glucoside have been compared in only three studies. Olthof et al. (2000) found that the pharmacokinetic profiles in human plasma were almost identical after ingestion of a single dose of either one of the glucosides. This is an indication that our in situ perfusion model is an appropriate model to study the small intestinal uptake of quercetin glucosides in human subjects. In accordance with our present study, both Gee et al. (2000) and Day et al. (2003) found that after incubation of an isolated rat intestinal segment with Q3glucoside and Q4'glucoside, serosal transfer and tissue concentrations of quercetin and its metabolites were similar for both glucosides. However, in their everted jejunal sac model mucosal hydrolysis of Q4' glucoside (represented as the appearance of free quercetin in the mucosal solution) was reported to be 10 times greater than Q3glucoside hydrolysis. In our present study, mucosal quercetin aglycone concentration was two times higher for $\mathrm{Q} 4^{\prime}$ glucoside than for Q3glucoside, while hydrolysis of $\mathrm{Q} 4^{\prime}$ glucoside (calculated as the decrease in glucoside concentration before and after perfusion) was only $5 \%$ greater than Q3glucoside hydrolysis. Thus, only the direct measurement of glucosides, but not the appearance of free quercetin, accurately reflects intestinal hydrolysis of flavonoid glucosides.

Quercetin is predominantly found glycosylated at the 3- or 4'-position, but a sugar can also be attached at other positions in the parent molecule, such as the 7hydroxyl group. Because no pure quercetin-7-glucoside could be obtained, we could not determine whether uptake is influenced by conjugation at this position. The limited data available for other flavonoids, however, do not suggest that the small intestinal uptake of a 7-glucoside would be much lower than the uptake of the aglycone, or the corresponding 3- or $4^{\prime}$-glucosides (Spencer et al. 1999; Felgines et al. 2000; Zubik \& Meydani, 2003). In addition, the substrate specificity of purified sheep LPH was no different for luteolin 7 - or $4^{\prime}$-glucoside, and hydrolysis of 7-glucosides of naringenin, eriodictyol and apigenin occurred at rates similar to those reported for Q3glucoside and Q4'glucoside (Németh et al. 2003).

In intact rats fed a single meal containing $66 \mu \mathrm{mol}$ Q3glucoside or Q3rhamnoside, plasma levels of quercetin metabolites were $33.2 \mu \mathrm{mol} / \mathrm{l}$ for the Q3glucoside group and not detectable for the Q3rhamnoside group at $4 \mathrm{~h}$ after the start of the meal (Morand et al. 2000). This is in line with our present findings. The intestinal absorption of Q3galactoside and Q3arabinoside has, to our knowledge, not been studied before. However, the substrate specificity of purified sheep LPH for several flavonoid 
glycosides has recently been published (Németh et al. 2003). These fully support our present data: Q3glucoside and Q4'glucoside are good substrates for LPH with hydrolysis rates of 3.16 and $3.08 \mu \mathrm{mol} / \mathrm{min}$ per $\mathrm{mg}$ protein respectively. Q3galactoside is a poor substrate (hydrolysis rate 0.13), and Q3rhamnoside and Q3arabinoside were not hydrolysed at all. This underlines the importance of LPH in the small intestinal uptake of flavonoid glycosides. Because LPH is traditionally considered a galactosidase, it was rather surprising to find that Q3galactoside was absorbed only to a very small extent, and that indeed these data are supported by the low hydrolysis rate recently reported by Németh et al. (2003). The only difference between Q3glucoside and Q3galactoside is the spatial configuration of the hydroxyl group at the $\mathrm{C} 4$ position of the sugar. This hydroxyl group seems to be a major determinant of the hydrolysis rate.

In intact mammals, digestion is facilitated by the secretion of enzymes in saliva and pancreatic juice. In the small intestinal perfusion model, the quercetin glycosides administered are not exposed to digestive juices, which can potentially limit the generalisability of results obtained with this model. The mammalian salivary glands and pancreas do not contain a ß-glycosidase, but they do secrete $\alpha$-amylase, an enzyme that catalyses the hydrolysis of $\alpha$-D-glucosidic linkages (Wong \& Robertson, 2003). Although Q3arabinoside is an $\alpha$-glycoside, it is unlikely that $\alpha$-amylase will hydrolyse the sugar because it has an L- and not a D-configuration. Thus, it is unlikely that in intact rats the Q3arabinoside would be absorbed in the small intestine.

Bile is generally considered an important excretion route for flavonoids. However, firm evidence to support this contention was thus far limited to one study. In line with our findings, Manach et al. (1996) reported high equimolar concentrations of quercetin, isorhamnetin and tamarixetin in the bile of rats fed quercetin $(2.5 \mathrm{~g} / \mathrm{kg}$ diet $)$ for $14 \mathrm{~d}$. The fact that both we and Manach et al. (1996) found no tamarixetin in plasma suggests that this metabolite is not formed in the intestine, but only in the liver and immediately excreted into bile. The high methylated:unmethylated quercetin derivative ratio in bile (approximately 2.0) compared with plasma $(0 \cdot 2)$ suggests that methylation of quercetin strongly favours biliary excretion. Extremely efficient clearance of tamarixetin from plasma is therefore an alternative explanation. However, this is not likely to explain the complete absence of tamarixetin from portal vein plasma. Interestingly, in the study of Manach et al. (1996) bile concentrations of quercetin metabolites were similar to those in our present study, but plasma levels were much greater. In their $14 \mathrm{~d}$ study with extremely high doses of quercetin, adaptive mechanisms in intestinal or hepatic metabolism and transport, or in the large intestinal microflora, may have occurred; these did not take place in our present short-term study. Once absorption of the quercetin glycosides has taken place, metabolite pattern and excretion rate into bile is no longer dependent on the type of glycoside administered.

Thus, our present data are supportive of previous data showing that hydrolysis of the sugar moiety is a necessary step for absorption of quercetin glycosides in the small intestine to occur. Of several glycosides tested in our present study, only the 3 -glucoside and the $4^{\prime}$-glucoside were absorbed in substantial quantities. The position of the glucose moiety (3 or $4^{\prime}$ ) did not influence absorption. Excretion patterns into bile were similar for quercetin glycosides with similar absorption characteristics. Although it is commonly stated that glucose is the sugar most frequently found in flavonol glycosides, two of the three major sources of flavonols in the Netherlands, tea and apple (Hertog et al. 1993), contain only small amounts of flavonol glucosides. In apple, the major quercetin glycosides are the galactoside, rhamnoside, arabinoside and xyloside, with only $100 \mathrm{~g}$ glucoside/kg quercetin glycosides. In tea the glucoside concentration is somewhat greater, but still only $250 \mathrm{~g} / \mathrm{kg}$ quercetin glycosides (Hollman \& Arts, 2000). Our present data show that the bioavailability of important dietary quercetin glycosides is very poor. This has implications for the in vivo bioactivity that can be expected after oral intake of these compounds. Findings from the present study underline the importance of compiling food composition tables with individual glycosides rather than aglycones only, and are relevant for manufacturers and consumers considering the production and use of quercetin-enriched functional foods or supplements.

\section{Acknowledgements}

Dini Venema is gratefully acknowledged for analytical support. The study was supported by grant QLK1-CT1999-00505 from the European Community, Framework V Programme (POLYBIND), and by the Dutch Ministry of Agriculture, Nature Management and Fisheries.

\section{References}

Arts ICW, Venema DP \& Hollman PCH (2003) Quantitative determination of flavonols in plant foods and biological fluids. In Methods in Polyphenol Analysis, pp. 214-228 [C Santos-Buelga and G Williamson, editors]. Cambridge: The Royal Society of Chemistry.

Crespy V, Morand C, Besson C, Manach C, Demigne C \& Remesy C (2001) Comparison of the intestinal absorption of quercetin, phloretin and their glucosides in rats. J Nutr 131, 2109-2114.

Day AJ, Bao YP, Morgan MRA \& Williamson G (2000a) Conjugation position of quercetin glucuronides and effect on biological activity. Free Radic Biol Med 29, 1234-1243.

Day AJ, Canada FJ, Diaz JC, Kroon PA, Mclauchlan R, Faulds CB, Plumb GW, Morgan MRA \& Williamson G (2000b) Dietary flavonoid and isoflavone glycosides are hydrolysed by the lactase site of lactase phlorizin hydrolase. FEBS Lett 468, 166-170.

Day AJ, Gee JM, DuPont MS, Johnson IT \& Williamson G (2003) Absorption of quercetin-3-glucoside and quercetin-4'glucoside in the rat small intestine: the role of lactase phlorizin hydrolase and the sodium-dependent glucose transporter. Biochem Pharmacol 65, 1199-1206.

Day AJ \& Williamson G (2001) Biomarkers for exposure to dietary flavonoids: a review of the current evidence for identification of quercetin glycosides in plasma. Br J Nutr 86, Suppl 1, 105-110. 
Felgines C, Texier O, Morand C, Manach C, Scalbert A, Regerat F $\&$ Remesy C (2000) Bioavailability of the flavanone naringenin and its glycosides in rats. Am J Physiol Gastrointest Liver Physiol 279, G1148-G1154.

Gee JM, DuPont MS, Day AJ, Plumb GW, Williamson G \& Johnson IT (2000) Intestinal transport of quercetin glycosides in rats involves both deglycosylation and interaction with the hexose transport pathway. J Nutr 130, 2765-2771.

Graefe EU, Wittig J, Mueller S, Riethling AK, Uehleke B, Drewelow B, Pforte H, Jacobasch G, Derendorf H \& Veit M (2001) Pharmacokinetics and bioavailability of quercetin glycosides in humans. J Clin Pharmacol 41, 492-499.

Hertog MGL, Hollman PCH, Katan MB \& Kromhout D (1993) Intake of potentially anticarcinogenic flavonoids and their determinants in adults in the Netherlands. Nutr Cancer 20, 21-29.

Hollman PC, Buijsman MN, van Gameren Y, Cnossen EP, de Vries JH \& Katan MB (1999) The sugar moiety is a major determinant of the absorption of dietary flavonoid glycosides in man. Free Radic Res 31, 569-573.

Hollman PCH \& Arts ICW (2000) Flavonols, flavones and flavanols - nature, occurrence and dietary burden. J Sci Food Agric 80, 1081-1093.

Hollman PCH, van Trijp JMP, Buysman MNCP, van der Gaag MS, Mengelers MJB, de Vries JHM \& Katan MB (1997) Relative bioavailability of the antioxidant flavonoid quercetin from various foods in man. FEBS Lett 418, 152-156.

Lommen A, Godejohann M, Venema DP, Hollman PCH \& Spraul M (2000) Application of directly coupled LC-NMR-MS for the identification and confirmation of quercetin glycosides and phloretin glycosides in apple peel. Anal Chem 72, 1793-1797.

Manach C, Morand C, Crespy V, Demigne C, Texier O, Regerat F \& Remesy C (1998) Quercetin is recovered in human plasma as conjugated derivatives which retain antioxidant properties. FEBS Lett 426, 331-336.

Manach C, Texier O, Regerat F, Agullo G, Demigne C \& Remesy C (1996) Dietary quercetin is recovered in rat plasma as conjugated derivatives of isorhamnetin and quercetin. J Nutr Biochem 7, 375-380.

Morand C, Manach C, Crespy V \& Remesy C (2000) Quercetin
3-O-beta-glucoside is better absorbed than other quercetin forms and is not present in rat plasma. Free Radic Res 33, $667-676$.

Németh K, Plumb GW, Berrin JG, Juge N, Jacob R, Naim HY, Williamson G, Swallow DM \& Kroon PA (2003) Deglycosylation by small intestinal epithelial cell beta-glucosidases is a critical step in the absorption and metabolism of dietary flavonoid glycosides in humans. Eur J Nutr 42, 29-42.

Nijveldt RJ, van Nood E, van Hoorn DEC, Boelens PG, van Norren K \& van Leeuwen PAM (2001) Flavonoids: a review of probable mechanisms of action and potential applications. Am J Clin Nutr 74, 418-425.

Olthof MR, Hollman PCH, Vree TB \& Katan MB (2000) Bioavailabilities of quercetin-3-glucoside and quercetin-4'-glucoside do not differ in humans. J Nutr 130, 1200-1203.

Rice-Evans CA, Miller NJ \& Paganga G (1996) Structure-antioxidant activity relationships of flavonoids and phenolic acids. Free Radic Biol Med 20, 933-956.

Sesink ALA, Arts ICW, Faassen-Peters M \& Hollman PCH (2003) Intestinal uptake of quercetin-3-glucoside in rats involves hydrolysis by lactase phlorizin hydrolase. $J$ Nutr 133, 773-776.

Spencer JP, Chowrimootoo G, Choudhury R, Debnam ES, Srai SK \& Rice-Evans C (1999) The small intestine can both absorb and glucuronidate luminal flavonoids. FEBS Lett 458, 224-230.

Stahl W, van den Berg H, Arthur J, et al. (2002) Bioavailability and metabolism. Mol Aspects Med 23, 39-100.

Verhoeyen ME, Bovy A, Collins G, Muir S, Robinson S, de Vos CH \& Colliver S (2002) Increasing antioxidant levels in tomatoes through modification of the flavonoid biosynthetic pathway. J Exp Bot 53, 2099-2106.

Wong DWS \& Robertson GH (2003) Alpha-amylases. In Handbook of Food Enzymology, pp. 707-718 [JR Whitaker, AGJ Voragen and DWS Wong, editors]. New York: Marcel Dekker, Inc.

Zubik L \& Meydani M (2003) Bioavailability of soybean isoflavones from aglycone and glucoside forms in American women. Am J Clin Nutr 77, 1459-1465. 